\title{
Prevalence of stress and depression and associated factors among women seeking a first- trimester induced abortion in China: A cross- sectional study
}

\section{Qiuxiang Zhang}

Peking University People's Hospital Department of Gynaecology and Obstetrics

Na Wang (D na_wang_3@163.com)

Capital Medical University School of Nursing https://orcid.org/0000-0003-4310-8911

Yanchu Hu

Peking University School of Nursing

\section{Debra K. Creedy}

Griffith University School of Nursing and Midwifery

\section{Research Article}

Keywords: Abortion, Mental Health, Perceived Stress, Depression, Intimate Relationship, Domestic violence, Resilience, Social support, China

Posted Date: January 27th, 2022

DOI: https://doi.org/10.21203/rs.3.rs-1234135/v1

License: (1) This work is licensed under a Creative Commons Attribution 4.0 International License.

Read Full License

Version of Record: A version of this preprint was published at Reproductive Health on March 9th, 2022. See the published version at https://doi.org/10.1186/s12978-022-01366-1. 


\section{Abstract \\ Objectives}

To determine the prevalence of stress and depression and associated factors among women seeking a first-trimester induced abortion in China.

\section{Methods}

A cross-sectional study was conducted in a tertiary hospital in Beijing, from April 1st to Oct 31st, 2021. Women seeking termination of an intrauterine first-trimester pregnancy were invited to participate and complete a digital self-administered questionnaire. The survey included socio-demographic and health questions, Perceived Stress Scale-10 (PSS-10), and Patient Health Questionnaire-9 (PHQ-9). Descriptive analyses and binary logistic regression analyses were performed using SPSS 23.0.

\section{Results}

A total of 253 women participated. Prevalence of high perceived stress (cut-off $\geq 20$ ) and depressive symptoms (cut-off $\geq 10$ ) was $25.3 \%$ and $22.5 \%$ respectively. Women were more likely to suffer high stress if they reported low resilience $(\mathrm{aOR}=16.84,95 \% \mathrm{Cl} 5.18-54.79)$, were not-using contraceptives $(\mathrm{aOR}=3.27,95 \% \mathrm{Cl} 1.39-6.29)$, had low social support $(\mathrm{aOR}=2.95,95 \% \mathrm{Cl} 1.39-6.29)$, were non-local residents $(\mathrm{aOR}=2.51,95 \% \mathrm{Cl} 1.15-5.92)$, were dissatisfied with their intimate relationship $(\mathrm{aOR}=2.44$, $95 \% \mathrm{Cl} 1.15-5.16)$, or held pro-life attitudes towards abortion $(\mathrm{aOR}=1.04,95 \% \mathrm{Cl} 1.18-3.53)$. Odds of experiencing depression were higher among women who also reported high perceived stress $(\mathrm{aOR}=$ 19.00, 95\% Cl 7.67-47.09), had completed higher education ( $\mathrm{aOR}=12.28,95 \% \mathrm{Cl} 1.24-121.20)$, and were non-local residents $(\mathrm{aOR}=3.38,95 \% \mathrm{Cl} 1.37-8.32)$.

\section{Conclusions}

The magnitude of perceived stress and depression was high among Chinese women seeking a firsttrimester induced abortion. It is necessary to comprehensively evaluate the mental health of all women seeking an abortion. Interventions to mitigate relevant associated factors could improve the psychological wellbeing of women.

\section{Plain Language Summary}

Abortion is a stressful life event. Understanding how women perceive having an abortion and their psychosocial responses will enable health providers to understand women's needs and provide highquality care. This study aims to determine the frequency of stress and depression among women seeking an induced abortion in early pregnancy in China and associated factors Around $25.3 \%$ of women reported 
high stress and $22.5 \%$ reported depression. Women were more likely to suffer high stress if they reported low resilience, were not-using contraceptives, had low social support, were non-local residents, were unhappy with their intimate relationship, or held pro-life attitudes towards abortion. High perceived stress, high education level, and non-local status were associated with depression. The findings indicate stress and depression are quite common among women seeking an abortion in China, especially those with high-risk factors. It is necessary to evaluate and promote the mental health of women seeking an abortion.

\section{Background}

Around 73 million abortions are performed annually worldwide [1], and more than $90 \%$ occur in the firsttrimester [2]. A single first-trimester abortion is physically safe when conducted using WHO-recommended methods by qualified professionals [3]. However, in most cases, an abortion is a stressful life event, that happens in the context of an unwanted/unintended pregnancy and poses challenges to women psychological wellbeing [4]. While some women cope well, others suffer a range of mental health consequences $[4,5]$.

Negative associations between perceived stress and psychological health have been observed across a wide variety of studies conducted among women having a preterm birth [8] and those experiencing a miscarriage [9]. Understanding women's perceptions of coping with abortion, as well as associated factors, can be helpful for health service providers to assess and plan for individual variation in coping. Various models attempt to explain variability in a person's response to a stressful life event. The Transactional Model derived from stress and coping theories [4, 6], for example, can help to explain factors affecting women's response to an abortion experience. According to this model, a woman's psychological experience is shaped by how she appraises the significance of the abortion and her ability to cope [7]. Perceived stress emerges from situations that women appraise as exceeding their ability or resources to cope [7]. Women's perceptions of stress can influence their choice of specific coping behaviours and subsequent psychological well-being [7].

Few studies, however, have reported perceptions of stress among women seeking an abortion. Using the 4-item Perceived Stress Scale (PSS-4), Harris et al [10] revealed minimal stress among 254 women seeking first-trimester abortions in the U.S. (mean of 4.2 on a 0-16 scale). Another study also conducted in the U.S. reported an average PSS-4 score of 7.24 among 784 women at the time of abortion seeking but placed no limitation on gestational age [11]. Variability in findings may be related to heterogeneity in women's gestation and reliability of the PSS-4. A systematic review comparing psychometric properties (e.g. internal consistency reliability, factorial validity, and hypothesis validity) of all three versions of the PSS $(4,10$, and 14 items) recommended the PSS-10 to measure perceived stress [12]. Yet, the PSS-10 is rarely used to explore individuals' perceptions of stress associated with abortion.

Studies on other psychological responses of women to abortion have also reported significantly different outcomes [4]. Prevalence of depression has ranged from $7.9 \%$ 53.5\% [13, 14]. In addition to 
heterogeneity in measures and evaluation timeframe, few studies controlled for possible confounders [5]. Possible confounders were identified by the American Psychological Association (APA) Task Force on Mental Health and Abortion (TFMHA) [4] in a review of 23 studies conducted among U.S. women. Factors such as a prior history of mental health problems, gestational age, low self-esteem, low resilience, low perceived ability to cope with the abortion, ambivalence about the abortion decision, stigma, and low social support were negatively associated women's abortion psychological experiences.

Another potential factor impacting on women's psychological outcomes relates to the wider sociopolitical context within which pregnancy and abortion occur. General statements about the impact of abortion on women psychological wellbeing can be inaccurate, due to the regional differences in legal context, cultural views, as well as associated social stigma of abortion [4, 5]. In 2011, the UK National Collaborating Centre for Mental Health (NCCMH) systematically reviewed a broader international literature on abortion [5]. Among the 27 studies included, only two were conducted in a developing country (South Africa and Russia), with 13 studies conducted in the U.S. and six in Northern Europe [5]. None of the studies had been conducted among the Chinese population.

In China approximately 6-9 million induced abortions are performed every year, corresponding to $15 \%$ of the world's total abortion numbers [15]. China has some of the most liberal abortion laws in the world, which allow first-trimester abortion without restriction as to the reason, with the exception of sex-selective abortions which are prohibited [16]. According to the State of World Population 2018 (UNFPA), many Chinese women report a continued desire for only one child [16], even though the One-Child Policy ended in 2013. Despite legislation that supports women's reproductive rights and access to safe abortion services, little is known about how Chinese women perceive having an abortion and their psychosocial responses at the time of abortion-seeking. Furthermore, there is no published evidence regarding factors associated with individual variation in women's psychological well-being.

\section{Objectives}

This study aimed to 1) determine the prevalence of high perceived stress and depression among women seeking a first-trimester induced abortion in China; and 2) identify factors associated with high perceived stress and depression to help detect high-risk groups.

\section{Methods}

\section{Study design and setting}

A cross-sectional study was conducted in the Family Planning and Reproductive Health Centre (FPRHC) of a tertiary hospital in Beijing, China. Participants were recruited from the outpatient unit, where abortionrelated services are normally provided.

\section{Participants}


Women meeting the following criteria were invited to participate: 1) Chinese citizens or living in China; 2) could speak, read, and write in Chinese; 3) 18 years or older; 4) seeking termination of an intrauterine pregnancy; 5) less than 12 gestational weeks; and 6) certain about their abortion decision which was of their own free will. Women were excluded if they were: 1) presenting for a post-abortion follow-up examination, secondary treatment of an incomplete abortion, or non-abortion related services; 2) had been infected by COVID-19; 3) had a mental health history; or 4) unable to give informed consent (e.g., had severe intellectual disability).

\section{Sample size determination}

To determine prevalence of high perceived stress and depression, samples of 202 and 248 respectively were needed using a single population proportion formula, rate for high perceived stress of $p=24.7 \%$ [17] and rate for depression of $p=22.4 \%$ [18], $95 \% \mathrm{Cl}, 5 \%$ margin error, and design effect at 2 . Hence, a sample size above 248 was deemed sufficient.

\section{Survey content and Data collection}

Demographic information included participants' age, marital status, education, religion, income, and were local or non-local residents as recorded in the Chinese household registration (hukou) system (determining eligibility for publicly-funded services and welfare [19]). Reproductive history included parity, abortion history, and number of living children. Current circumstances were women's gestational age, whether the pregnancy was intended, reasons for choosing an abortion, attitude toward abortion, parenthood plan, and contraception use.

\section{Primary outcomes}

\section{Perceived Stress}

The Chinese version of the Perceived Stress Scale-10 (PSS-10) [20] relates to how unpredictable, uncontrollable, and overloaded respondents perceive their current situation since first finding out about the pregnancy to now. Scores range from 0 to 40 with a score $\geq 20$ considered high perceived stress [18, 21]. In this study, participants were also asked to indicate any other specific sources of stress in this current pregnancy.

\section{Depression symptoms}

Depression symptoms were measured by the Patient Health Questionnaire-9 (PHQ-9) [22]. The nine items address symptoms associated with experiencing pleasure, feeling down, and self-esteem. Participants rate the severity of their depressive symptoms since pregnancy on a 4-point scale $(0=$ not at all; $1=$ several days; 2 = more than half the days; and 3 = nearly every day). Total scores range from 0 to 27 , with a score $\geq 10$ indicating depression. The tool has been translated and valid for use among the Chinese population [23].

\section{Potential associated factors}




\section{Domestic Violence}

Terms such as 'domestic violence', 'intimate partner violence', and 'violence against women' are used interchangeably in the literature. We used the Domestic Violence Screening Tool (DVST) recommended by the American College of Obstetricians and Gynecologists to detect women's experience of violence during the preceding year by an intimate partner [24]. Three questions relate to physical, mental or verbal, and sexual abuse within the past year. Any positive response indicates the presence of violence. The tool has been translated and is valid for use among Chinese women [25].

\section{Intimate Relationship Satisfaction}

The 4-item Couples Satisfaction Index CSI-4 [26] measures intimate relationship quality. The CSI-4 uses different response formats, with total scores ranging from 0 to 21 , with higher scores indicating greater satisfaction and scores below 13.5 suggesting notable relationship dissatisfaction. The Cronbach's a reliability of the translated version with Chinese woman is 0.88 [27].

\section{Perceived Social Support}

The Medical Outcomes Study Social Support Scale (MOSSS-5) [28] measured participants' perceptions of social support since their pregnancy. Each item is scored on a 5-point Likert scale and summed for a total score ranging from $5-25$. A total score between $5-16$ is deemed as low perceived social support. The MOSSS- 5 has adequate test-retest reliability (0.89) and internal consistency $(0.97)$ in Chinese population [29].

\section{Resilience}

The Chinese version of the Brief Resilience Scale (BRS) [30], includes six statements about one's selfefficacy in dealing with stressful events on a scale of $1=$ strongly disagree to $5=$ strongly agree. Possible mean scores of all six-items are interpreted as low (1 - 2.99), normal (3 - 4.30), and high resilience (4.31 5) [31]. The Chinese version BRS has good reliability and internal consistency [32].

\section{Data collection}

A digital self-administered questionnaire was used to collect data. Two nurses employed by the center screened all women based on inclusion and exclusion criteria. After informed consent, eligible women completed the questionnaire on a tablet computer offered by the research team or on their own tablet by scanning a QR code. All participants were offered an optional private room as well as assistance from the first author to complete the questionnaire. Clinic nurses and the first author were trained for scientific research data collection.

\section{Data analysis}

All data were analyzed using Statistical Package for the Social Sciences (SPSS) ® version 23 [33]. Descriptive statistics were presented using frequency counts and percentages (categorical variables) or mean \pm standard deviation (continuous variables). Relationships between categorized outcomes and 
explanatory variables were tested by Chi-square. Multivariate binary logistic regression analysis was performed with stress and depression as the dichotomous variables respectively. Factors with $p$-value $<0.1$ were included in the binary logistic regression model, and $p$-values $<0.05$ were considered significant.

\section{Results}

\section{Characteristics of participants}

In total, 253 eligible women finished the survey with a response rate of $81.3 \%(253 / 311)$. The mean age of participants was 32.08 \pm 6.09 , with a predominant age group of 25-34 (43.5\%) (as shown in Table 1). Nearly two-thirds (64.4\%) were married, and half lived locally. Most women (99.2\%) were atheists, and $89.7 \%$ held pro-choice attitudes toward abortion. Around half (52.6\%) had children, with $45.5 \%$ having had an abortion before. The average parity was $2.13 \pm 0.85$ pregnancies. Most ( $82.2 \%)$ participants were less than nine weeks pregnant, with an average gestational age of $7.32 \pm 1.30$ weeks. Most women (87\%) indicated their pregnancy as unintended, and social factors were the most common cited reasons (63.2\%) for choosing an abortion. Only seven (2.8\%) women reported any type of IPV experience within the last year. However, 31.5\% indicated notable intimate relationship dissatisfaction (CSI-4<13.5). The mean score of perceived social support was $18.28 \pm 5.02$, with over a third (35.2\%) reporting low support (MOSSS-5<17). Participants reported a mean resilience score of $3.56 \pm 0.57$ with $9.5 \%$ reporting low resilience $(\mathrm{BRS}<3)$ 
Table 1

Demographic and reproductive characteristics of low and high perceived stress participants

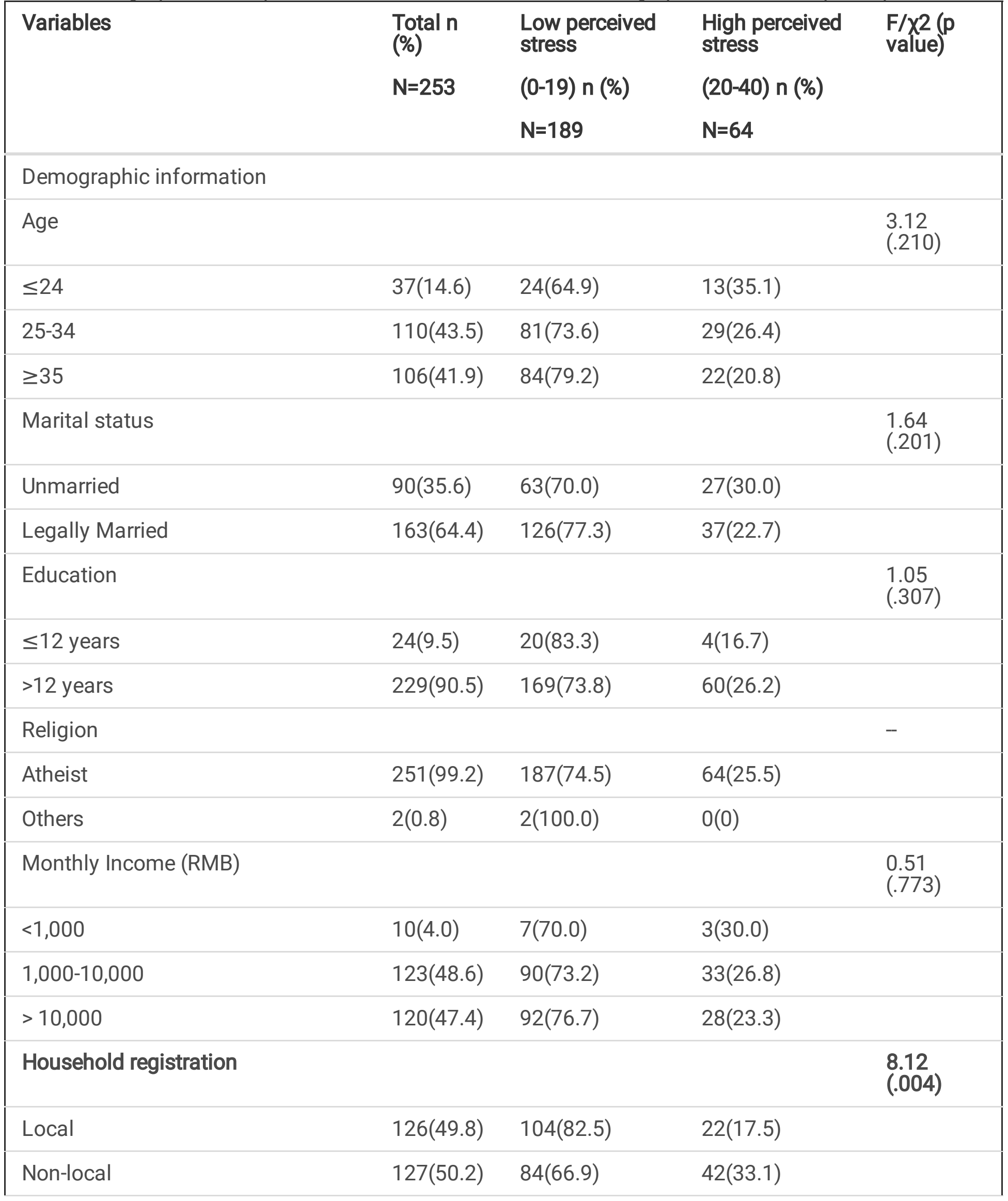




\begin{tabular}{|c|c|c|c|c|}
\hline \multirow[t]{3}{*}{ Variables } & $\begin{array}{l}\text { Total n } \\
\text { (\%) }\end{array}$ & $\begin{array}{l}\text { Low perceived } \\
\text { stress }\end{array}$ & $\begin{array}{l}\text { High perceived } \\
\text { stress }\end{array}$ & $\begin{array}{l}F / X 2(p \\
\text { value) }\end{array}$ \\
\hline & $\mathrm{N}=\mathbf{2 5 3}$ & $(0-19) n(\%)$ & $(20-40)$ n (\%) & \\
\hline & & $N=189$ & $N=64$ & \\
\hline Pre-existing physical condition & & & & $\begin{array}{l}0.12 \\
(.694)\end{array}$ \\
\hline No & $245(96.8)$ & 184(75.1) & $61(24.6)$ & \\
\hline Yes & $8(3.2)$ & $5(62.5)$ & $3(37.5)$ & \\
\hline \multicolumn{5}{|l|}{ Reproductive history } \\
\hline Parity & & & & $\begin{array}{l}9.44 \\
(.009)\end{array}$ \\
\hline 1 & 77(30.4) & $48(62.3)$ & $29(37.7)$ & \\
\hline 2 & $65(25.7)$ & $54(83.1)$ & 11(16.9) & \\
\hline$\geq 3$ & $111(43.9)$ & $87(78.4)$ & $24(21.6)$ & \\
\hline Previous abortion & & & & $\begin{array}{l}0.81 \\
(.369)\end{array}$ \\
\hline No & $138(54.5)$ & $100(72.5)$ & $38(27.5)$ & \\
\hline Yes & $115(45.5)$ & $89(77.4)$ & $26(22.6)$ & \\
\hline Existing Child(ren) & & & & $\begin{array}{l}6.27 \\
(.012)\end{array}$ \\
\hline No & $120(47.4)$ & $81(67.5)$ & $39(32.5)$ & \\
\hline Yes & $133(52.6)$ & 108(81.2) & 25(18.8) & \\
\hline \multicolumn{5}{|l|}{ Current status } \\
\hline Gestation weeks & & & & $\begin{array}{l}0.27 \\
(.601)\end{array}$ \\
\hline Mean & $7.32 \pm 1.30$ & & & \\
\hline$<9$ weeks & 208(82.2) & $154(74.0)$ & $54(26.0)$ & \\
\hline 9-12 weeks & $45(17.8)$ & $35(77.8)$ & $10(22.2)$ & \\
\hline Pregnancy intention & & & & $\begin{array}{l}0.08 \\
(.779)\end{array}$ \\
\hline Unintended & $220(87.0)$ & $165(75.0)$ & $55(25.0)$ & \\
\hline Intended & 33(13.0) & $24(72.7)$ & $9(27.3)$ & \\
\hline
\end{tabular}




\begin{tabular}{|c|c|c|c|c|}
\hline \multirow[t]{3}{*}{ Variables } & $\begin{array}{l}\text { Total } \mathbf{n} \\
\text { (\%) }\end{array}$ & $\begin{array}{l}\text { Low perceived } \\
\text { stress }\end{array}$ & $\begin{array}{l}\text { High perceived } \\
\text { stress }\end{array}$ & $\begin{array}{l}F / X 2(p \\
\text { value) }\end{array}$ \\
\hline & $\mathrm{N}=\mathbf{2 5 3}$ & $(0-19)$ n (\%) & $(20-40)$ n (\%) & \\
\hline & & $N=189$ & $N=64$ & \\
\hline Abortion reason & & & & $\begin{array}{l}1.06 \\
(.590)\end{array}$ \\
\hline Health-related & $93(36.8)$ & $68(73.1)$ & $25(26.9)$ & \\
\hline Social-related & $142(56.1)$ & $109(76.8)$ & $33(23.2)$ & \\
\hline Both & 18(7.1) & $12(66.7)$ & 6(33.2) & \\
\hline Opinion about abortion & & & & $\begin{array}{l}6.67 \\
(.010)\end{array}$ \\
\hline Pro-choice & 227(89.7) & 175(77.1) & $52(22.9)$ & \\
\hline Pro-life & $26(10.3)$ & 14(53.8) & $12(46.2)$ & \\
\hline Parenthood plan & & & & $\begin{array}{l}3.47 \\
(.176)\end{array}$ \\
\hline Yes & $35(13.8)$ & $24(68.6)$ & 11(31.4) & \\
\hline Current no, but yes in 2 years & $59(23.3)$ & $40(67.8)$ & 19(32.2) & \\
\hline No & $159(62.8)$ & 125(78.6) & $34(21.4)$ & \\
\hline Contraception use & & & & $\begin{array}{l}5.02 \\
(.025)\end{array}$ \\
\hline Yes & $209(82.6)$ & $162(77.5)$ & $47(22.5)$ & \\
\hline No & $44(17.4)$ & $27(61.4)$ & 17(38.6) & \\
\hline Domestic violence experience & & & & $\begin{array}{l}0.00 \\
(1.000)\end{array}$ \\
\hline No & $246(97.2)$ & $184(74.8)$ & $62(25.2)$ & \\
\hline Yes & $7(2.8)$ & $5(71.4)$ & $2(28.6)$ & \\
\hline Low Resilience & & & & $\begin{array}{l}34.66 \\
(.001)\end{array}$ \\
\hline Yes & $24(9.5)$ & $6(25.0)$ & 18(75.0) & \\
\hline No & $229(90.5)$ & 183(79.9) & $46(20.1)$ & \\
\hline Low Social support & & & & $\begin{array}{l}28.05 \\
(.001)\end{array}$ \\
\hline Yes & $89(35.2)$ & $49(55.1)$ & $40(44.9)$ & \\
\hline
\end{tabular}




\begin{tabular}{|c|c|c|c|c|}
\hline \multirow[t]{3}{*}{ Variables } & $\begin{array}{l}\text { Total n } \\
(\%)\end{array}$ & $\begin{array}{l}\text { Low perceived } \\
\text { stress }\end{array}$ & $\begin{array}{l}\text { High perceived } \\
\text { stress }\end{array}$ & $\begin{array}{l}F / X 2(p \\
\text { value) }\end{array}$ \\
\hline & $\mathrm{N}=253$ & $(0-19) n(\%)$ & $(20-40)$ n (\%) & \\
\hline & & $\mathrm{N}=189$ & $N=64$ & \\
\hline No & 164(64.8) & $140(85.4)$ & $24(14.6)$ & \\
\hline $\begin{array}{l}\text { Notable Intimate relationship } \\
\text { dissatisfaction }\end{array}$ & & & & $\begin{array}{l}14.64 \\
(.001)\end{array}$ \\
\hline Yes $<13.5$ & $92(36.4)$ & $56(60.9)$ & $36(39.1)$ & \\
\hline No & $161(63.6)$ & 133(82.6) & $28(17.4)$ & \\
\hline
\end{tabular}

Insert Table 1 about here

\section{Perceived stress and associated factors}

Of the 253 responses, the mean perceived stress score was $16.96 \pm 0.44$, with an overall prevalence of high perceived stress (PSS $\geq 20$ ) at $25.3 \%$. Table 1 presents associations between participants' characteristics and perceived stress levels when seeking a first-trimester abortion. As shown in Table 2, the logistic regression model revealed that women who were non-local residents, had pro-life attitudes towards abortion, did not use contraception, and had low resilience, low perceived social support, and relationship dissatisfaction were more likely to report high perceived stress. Women's resilience played the greatest role in perceived stress. The odds ratio of women with low resilience compared to those with high perceived stress was aOR $=16.84(95 \% \mathrm{Cl} 5.18-54.79, \mathrm{p}<0.001)$. 
Table 2

Factors associated with high perceived stress (PSS $\geq 20$ ) from binary logistic regression analysis

\begin{tabular}{|c|c|c|c|c|c|}
\hline Variable & $\begin{array}{l}\text { High perceived stress } \\
\mathrm{n}(\%)\end{array}$ & $\begin{array}{l}\text { B- } \\
\text { coefficient }\end{array}$ & $\mathrm{p}_{\text {value }}$ & aOR & $\begin{array}{l}95 \% \\
\mathrm{Cl}\end{array}$ \\
\hline \multicolumn{6}{|c|}{ Household registration } \\
\hline Local & $22(17.5)$ & & & 1.00 & \\
\hline Non-local & $42(33.1)$ & 0.918 & 0.020 & 2.51 & $\begin{array}{l}1.15- \\
5.92\end{array}$ \\
\hline Parity & & & 0.318 & & \\
\hline 1 & $29(37.7)$ & & & 1.00 & \\
\hline 2 & 11(16.9) & 0.668 & 0.286 & 1.95 & $\begin{array}{l}0.57- \\
6.65\end{array}$ \\
\hline$\geq 3$ & $24(21.6)$ & -0.155 & 0.775 & 0.86 & $\begin{array}{l}0.29- \\
2.49\end{array}$ \\
\hline \multicolumn{6}{|l|}{ Child } \\
\hline No & $25(32.5)$ & & & 1.00 & \\
\hline Yes & $39(18.8)$ & -0.273 & 0.620 & 0.76 & $\begin{array}{l}0.26- \\
2.24\end{array}$ \\
\hline \multicolumn{6}{|c|}{ Attitude toward abortion } \\
\hline Pro-choice & $52(22.9)$ & & & 1.00 & \\
\hline Pro-life & $12(46.2)$ & 0.714 & 0.010 & 2.04 & $\begin{array}{l}1.18- \\
3.53\end{array}$ \\
\hline \multicolumn{6}{|c|}{ Contraception use } \\
\hline Yes & $47(22.5)$ & & & 1.00 & \\
\hline No & $17(38.6)$ & 1.185 & 0.007 & 3.27 & $\begin{array}{l}1.39- \\
7.69\end{array}$ \\
\hline \multicolumn{6}{|c|}{ Low resilience } \\
\hline No & $46(20.1)$ & & & 1.00 & \\
\hline Yes & $18(75.0)$ & 2.824 & 0.000 & 16.84 & $\begin{array}{l}5.18- \\
54.79\end{array}$ \\
\hline \multicolumn{6}{|c|}{ Low social support } \\
\hline No & $24(14.6)$ & & & 1.00 & \\
\hline Yes & $40(44.9)$ & 1.081 & 0.005 & 2.95 & $\begin{array}{l}1.39- \\
6.29\end{array}$ \\
\hline
\end{tabular}




\begin{tabular}{|c|c|c|c|c|c|}
\hline Variable & $\begin{array}{l}\text { High perceived stress } \\
\mathrm{n}(\%)\end{array}$ & $\begin{array}{l}\text { B- } \\
\text { coefficient }\end{array}$ & $\begin{array}{l}\mathrm{p} \\
\text { value }\end{array}$ & aOR & $\begin{array}{l}95 \% \\
\mathrm{Cl}\end{array}$ \\
\hline \multicolumn{6}{|c|}{$\begin{array}{l}\text { Notable intimate relationship } \\
\text { dissatisfaction }\end{array}$} \\
\hline No & $28(17.4)$ & & & 1.00 & \\
\hline Yes (<13.5) & $36(39.1)$ & 0.892 & 0.020 & 2.44 & $\begin{array}{l}1.15- \\
5.16\end{array}$ \\
\hline
\end{tabular}

Specific sources of women's stress included emotional or mental stress from making or accepting the abortion decision (82.2\%), physical discomfort (78.7\%), concerns of potential complications from the abortion (34.8\%), intimate relationship dissatisfaction (28.9\%), and keeping the abortion a secret (25.3\%). Only $15 \%$ of women cited accessing abortion services as a stressor.

\section{Depression and associated factors}

The average PHQ-9 score was $6.96 \pm 4.90$ with the overall rate of depression (PHQ $\geq 10$ ) being $22.5 \%$. Women with depression tended to be highly educated $(p<0.05)$, non-local residents $(p<0.01)$, with low resilience $(p<0.01)$, low perceived social support $(p<0.01)$, relationship dissatisfaction $(p<0.01)$, or experiencing high perceived stress $(p<0.01)$ (Table 3$)$. The regression model revealed that depression was associated with high education level, non-local status, and high perceived stress (see supplementary file for detailed results). The adjusted odds ratio of factors contributing to depression were high perceived stress [aOR $=19.00,95 \% \mathrm{Cl} 7.67-47.09]$ compared to women with normal or low perceived stress; having more than 12 years of education [aOR $=12.28,95 \% \mathrm{Cl} 1.24-121.20$ ] compared with less educated women; and non-local residents $[\mathrm{aOR}=3.38,95 \% \mathrm{Cl} 1.37-8.32]$ compared with local residents. 
Table 3

Probable depression $(\mathrm{PHQ} \geq 10)$ rates stratified by participant characteristics

\begin{tabular}{|c|c|c|}
\hline Variables & Probable depression n (\%) & $F / \chi 2$ ( $p$ value) \\
\hline \multicolumn{3}{|l|}{ Demographic information } \\
\hline Age & & $4.41(.110)$ \\
\hline$\leq 24$ & $10(27.0)$ & \\
\hline $25-34$ & $30(27.3)$ & \\
\hline$\geq 35$ & $17(16.0)$ & \\
\hline Marital status & & $3.24(.072)$ \\
\hline Unmarried & $26(28.9)$ & \\
\hline Legally Married & $31(19.0)$ & \\
\hline Education $\#$ & & 5.12(.024) \\
\hline$\leq 12$ years & $1(4.2)$ & \\
\hline$>12$ years & $56(24.5)$ & \\
\hline Religion & & $0.00(1.000)$ \\
\hline Atheist & $57(22.7)$ & \\
\hline Others & $0(0)$ & \\
\hline Monthly Income (RMB) & & $1.99(.369)$ \\
\hline$<1,000$ & $1(10.0)$ & \\
\hline $1,000-10,000$ & $25(20.3)$ & \\
\hline$>10,000$ & $31(25.8)$ & \\
\hline Household registration & & $16.24(.000)$ \\
\hline Local & 15(11.9) & \\
\hline Non-local & $42(33.1)$ & \\
\hline Pre-existing physical condition & & $0.00(1.000)$ \\
\hline Yes & $2(25.0)$ & \\
\hline No & $55(22.4)$ & \\
\hline \multicolumn{3}{|l|}{ Reproductive history } \\
\hline Parity & & $5.07(.079)$ \\
\hline
\end{tabular}




\begin{tabular}{|c|c|c|}
\hline Variables & Probable depression n (\%) & $F / \chi 2$ ( $p$ value) \\
\hline 1 & $23(29.9)$ & \\
\hline 2 & $16(24.6)$ & \\
\hline$\geq 3$ & $18(16.2)$ & \\
\hline Previous Abortion & & $2.20(.138)$ \\
\hline Yes & 21(18.3) & \\
\hline No & $36(26.1)$ & \\
\hline Existing Child(ren) & & $2.34(.135)$ \\
\hline No & $32(26.7)$ & \\
\hline Yes & $25(18.8)$ & \\
\hline \multicolumn{3}{|l|}{ Current status } \\
\hline Gestation weeks & & $0.20(.650)$ \\
\hline$<9$ weeks & $48(23.1)$ & \\
\hline$\geq 9-12$ & $9(20.0)$ & \\
\hline Pregnancy intention & & $2.36(.125)$ \\
\hline Unintended & $53(24.1)$ & \\
\hline Intended & $4(12.1)$ & \\
\hline Abortion reason & & $0.56(.756)$ \\
\hline Health-related reasons & 19(20.4) & \\
\hline Social-related reasons & $33(23.2)$ & \\
\hline both & $5(27.8)$ & \\
\hline Opinion about abortion & & 2.43(.119) \\
\hline Pro-choice & $48(21.1)$ & \\
\hline Situationist or pro-life & $9(34.6)$ & \\
\hline Parenthood plan & & $3.63(.163)$ \\
\hline Yes & $11(31.4)$ & \\
\hline Current no, but yes in 2 years & 19(32.2) & \\
\hline No & $34(21.4)$ & \\
\hline Contraception & & $0.00(.972)$ \\
\hline
\end{tabular}




\begin{tabular}{|lll|}
\hline Variables & Probable depression $\mathbf{n}(\%)$ & F/X2 (p value) \\
\hline Yes & $47(22.5)$ & \\
\hline No & $10(22.7)$ & $\mathbf{0 . 0 0 ( 1 . 0 0 0 )}$ \\
\hline Domestic Violence & & \\
\hline Yes & $55(22.4)$ & $\mathbf{6 . 7 0 ( 0 . 1 0 )}$ \\
\hline Notable Intimate relationship dissatisfaction & $2(28.6)$ & \\
\hline Yes & $29(31.5)$ & $\mathbf{7 . 9 5 ( . 0 0 5 )}$ \\
\hline No & $28(17.4)$ & \\
\hline Low Social support & & $\mathbf{8 . 2 5}(.004)$ \\
\hline Yes & $29(32.6)$ & \\
\hline No & $28(17.1)$ & $\mathbf{8 4 . 6 7 ( . 0 0 0 )}$ \\
\hline Low Resilience & & \\
\hline Yes & $11(45.8)$ & \\
\hline No & $46(20.1)$ & \\
\hline High Perceived stress & $41(64.1)$ & \\
\hline Yes & $16(8.5)$ & \\
\hline No & & \\
\hline
\end{tabular}

\section{Discussion}

This study is one of the first to reveal high rates of perceived stress and depression among women seeking a first-trimester abortion in China. There was a consistent relationship between stress and depression. Women reported a relatively high level of stress compared to recent studies with the general public in China $[17,34]$. Similarly, depression symptoms were higher than recent research with the noninfected Chinese population during the COVID-19 pandemic [34, 35]. These differences may be due to the psychological and social challenges arising from having an unwanted pregnancy and the abortion process. An evidence-based review conducted by the UK National Collaborating Centre for Mental Health $(\mathrm{NCCMH})$ revealed an unwanted pregnancy and its resolution (abortion or give birth) often challenge women's emotional wellbeing (contributing to negative emotions, stress, and mental disorders) [5].

The high rates of stress and depression among women seeking an abortion may also be affected by the influence of gender on coping [36]. For example, when facing similar stressful life events, women compared to men, tend to feel greater perceptions of threat, less control, and less social support [37]. 
Similarly, coping behaviours of women tend to be more cautious and avoidance focused, while men use emotional inhibition and detachment [36]. Women are also more likely to be dissatisfied with their intimate relationship than men [38], or experience more disadvantage as a migrant than their male counterparts [39]. These findings indicate the necessity for further investment in evaluating and promoting the mental health of women when seeking abortion, particularly among those with high-risk factors.

We found perceptions of high stress were associated with low resilience, not using contraceptives, low social support, non-local status, intimate relationship dissatisfaction, or pro-life attitudes towards abortion. Depression was associated with high perceived stress, high levels of education, and non-local status. These risk factors are congruent with a report on mental health and abortion by the APA Task Force [4]. Similar environmental factors (e.g., problematic intimate relationships, low social support, or exposure to other stressful life events) and personal characteristics (e.g., low resilience low self-efficacy, or problematic health behaviours) were identified [4]. Previous intervention studies suggest that women's resilience [40], use of contraceptives [41], perceptions of social support [42], as well as intimate relationship satisfaction [38] are modifiable. For example, a systematic review and meta-analysis found cognitive behavioral therapy and interventions using mindfulness techniques can effectively improve individual resilience [40]. Another systematic review concluded that pre-abortion counselling was effective in promoting contraception use if accompanied by the provision of contraceptive methods [41]. Couples counselling or male partner involvement were also helpful [41].

The current study identified factors that are less amenable to change (like being a migrant, or holding prolife attitudes towards abortion), but are meaningful to clinicians when assessing women's vulnerability in order to provide comprehensive evaluation and timely support to women in high-risk groups [4].

In our study, only $2.8 \%$ of women reported experiencing violence in the preceding year, which was distinctly lower than rates $(30-62 \%)$ reported in previous studies using similar brief self-report DV screening tools with women seeking an abortion [43,44]. However, our study revealed a high level of intimate relationship dissatisfaction (31.5\%) which is similar to existing studies [45]. Previous studies reveal that DV is closely related to relationship dissatisfaction $[46,47]$. The observed unexpected disagreement between low DV experience and high relationship dissatisfaction in our study suggests the possibility of DV underreporting. It is possible that asking straightforward DV questions was insensitive or culturally inappropriate to detecting abuse among Chinese women seeking an abortion. Developing a trusting relationship with a known care provider is more likely to encourage disclosure from women about relationship violence.

\section{Limitations}

Our findings should be interpreted in light of several limitations. Participating women were all from one Chinese metropolitan abortion clinic and most were atheists. The recruited sample may therefore not be representative of women in the wider community or women who practice their religious faith. The

Page $17 / 24$ 
sensitive nature of studying the psychological status of Chinese women seeking a first-trimester induced abortion may have contributed to the under-reporting of some indicators like DV experience or abortion history.

Importantly, the study was conducted during the COVID-19 pandemic, even though only a small number of COVID-19 cases existed in Beijing during the survey period [48], and study participants were limited to those who were not infected or did not have a COVID-19 infection history. The enforcement of COVID related measures such as closing borders or mandatory quarantine could potentially impact women's daily activities [49], the support resources at their disposal [50], as well as their actual abortion experience and associated psychological responses [51]. To determine the generalizability of our findings, we subsequently compared demographic and reproductive characteristics of women included in this study to a national cross-sectional study of 79,954 women in China who had an induced abortion in 2013 (preCOVID). The analysis revealed that women aged $25-34$ years represented $53.1 \%$ of the pre-COVID group versus $43.5 \%$ among this study group $(0.01<p<0.05)$. Marital status was similar $(68.2 \%$ in the pre-COVID group vs $64.4 \%, p>0.05)$ as were the proportion of women who had children $(57.3 \%$ pre-COVID vs $52.6 \%$, $p>0.05$ ) [52]. The mean gestational age of participating women was $43.7 \pm 6.2$ days $\sim 59.0 \pm 14.5$ days in previously published studies [53-55], compared to $51 \pm 7.3$ days in the current study. Variation in the characteristics of women accessing abortion services pre- and post- COVID was not observed from this comparison. Future studies should, however, specifically investigate the impact of COVID on women's abortion seeking behavior as well as their abortion experience.

\section{Conclusions}

This study found a high prevalence of perceived stress and depression among Chinese women seeking a first-trimester induced abortion. Related risk factors of high perceived stress were low resilience, not-using contraceptives, low social support, non-local status, intimate relationship dissatisfaction, and holding prolife attitudes towards abortion. High perceived stress, high education level, and non-local status were consistently associated with depression. Results reveal the necessity for evaluating the mental health of all women seeking an abortion. The identified risk factors can be used in screening high-risk groups and promoting the implementation of targeted interventions in abortion services to improve the psychological wellbeing of women.

\section{Abbreviations}




\begin{tabular}{ll} 
APA & American Psychological Association \\
\hline BRS & Brief Resilience Scale \\
\hline COVID-19 & Corona Virus Disease in 2019 \\
\hline CSI-4 & Couples Satisfaction Index (4-item) \\
\hline DV & Domestic Violence \\
\hline DVST & Domestic Violence Screening Tool \\
\hline FPRHC & Family Planning and Reproductive Health Centre \\
\hline IPV & Intimate Partner Violence \\
\hline MOSSS-5 & Medical Outcomes Study Social Support Scale \\
\hline NCCMH & National Collaborating Centre for Mental Health \\
\hline OR & Odds ratio \\
\hline PHQ & Patient Health Questionnaire \\
\hline PSS & Perceived Stress Scale-10 \\
\hline QR code & Quick Response Code \\
\hline SPSS & Statistical Package for the Social Sciences \\
\hline TFMHA & Task Force on Mental Health and Abortion \\
\hline WHO & World Health Organization \\
\hline
\end{tabular}

\section{Declarations}

\section{Ethics approval and consent to participate}

This study was approved by the Institutional Ethical Review Boards of Peking University People's Hospital (2021PHB003-001) in March 2021. Written consent was obtained from all participants.

\section{Consent for publication}

Written consent for publication has been obtained from all participants during the informed consent process.

\section{Availability of data and materials}


Research data are only available on request from the corresponding author due to ethical restrictions and policy concerns.

\section{Competing interests}

All authors declare no competing interests, or other interests that might be perceived to influence the results and/or discussion reported in this paper.

\section{Funding}

None

\section{Authors' contributions}

QZ and NW contributed equally to this work. NW and YC contributed to the conceptualization, design, and interpretation of the study. QZ and NW collected data and wrote the first draft. DKC read and provided comments and wrote revisions on subsequent drafts of the manuscript. All authors approved the final version.

\section{Acknowledgments}

We fully understand sharing an abortion experience requires courage and self-giving, and we would like to express our highest respect and deepest appreciation to all the women participating in the research. We would also like to acknowledge the clinicians involved in the study for their invaluable contribution to data collection and expert support.

\section{References}

1. Bearak, J., et al., Unintended pregnancy and abortion by income, region, and the legal status of abortion: estimates from a comprehensive model for 1990-2019. The Lancet Global Health, 2020. 8(9): p. e1152-e1161.

2. Kortsmit, K., et al., Abortion Surveillance-United States, 2018. MMWR Surveillance Summaries, 2020. 69(7): p. 1.

3. Singh, S. and I. Maddow-Zimet, Facility-based treatment for medical complications resulting from unsafe pregnancy termination in the developing world, 2012: a review of evidence from 26 countries. International Journal of Obstetrics and Gynaecology, 2016. 123(9): p. 1489-98.

4. American Psychological Association, Report of the Task Force on Mental Health and Abortion. 2008, Task Force on Mental Health and Abortion: Washington, DC, USA. 
5. National Collaborating Centre for Mental Health, U., Induced abortion and mental health: $A$ systematic review of the mental health outcomes of induced abortion, including their prevalence and associated factors. London: Academy of Medical Royal Colleges, 2011.

6. Cohen, S.W., T.A., Stress, social support, and the buffering hypothesis. Psychological Bulletin Journal, 1985. 98(2): p. 310-357.

7. Wethington, E., K. Glanz, and M.D. Schwartz, Stress, Coping, and Health Behavior, in Health behavior: Theory, research, and practice. , K. Glanz, B.K. Rimer, and K. Viswanath, Editors. 2015. p. 223-242.

8. Staneva, A., et al., The effects of maternal depression, anxiety, and perceived stress during pregnancy on preterm birth: A systematic review. Women and Birth, 2015. 28(3): p. 179-193.

9. Chen, S.L., et al., Stress, anxiety and depression perceived by couples with recurrent miscarriage. International journal of nursing practice, 2020. 26(2): p. e12796.

10. Harris, L.F., et al., Perceived stress and emotional social support among women who are denied or receive abortions in the United States: a prospective cohort study. BMC women's health, 2014. 14(1): p. 76.

11. Biggs, M.A., et al., Developing and validating the Psychosocial Burden among people Seeking Abortion Scale (PB-SAS). PloS one, 2020. 15(12): p. e0242463.

12. Lee, E.-H., Review of the psychometric evidence of the perceived stress scale. Asian nursing research, 2012. 6(4): p. 121-127.

13. Moafi, F., et al., Spiritual intelligence and post-abortion depression: A coping strategy. Journal of religion and health, 2021. 60(1): p. 326-334.

14. Steinberg, J.R. and L.B. Finer, Examining the association of abortion history and current mental health: A reanalysis of the National Comorbidity Survey using a common-risk-factors model. Social Science \& Medicine, 2011. 72(1): p. 72-82.

15. National Health and Family Planning Commission of the People's Republic of China, China's Health and Family Planning Statistical Yearbook. 2016, Peking Union Medical College Press: Beijing, China.

16. UNFPA, et al., The power of choice: reproductive rights and the demographic transition - state of world population. 2018.

17. Chen, $\mathrm{H}_{\text {., }}$ et al., Collective self-esteem and perceived stress among the non-infected general public in China during the 2019 coronavirus pandemic: A multiple mediation model. Personality and Individual Differences, 2021. 168: p. 110308.

18. Gokoel, A.R., et al., Influence of perceived stress on prenatal depression in Surinamese women enrolled in the CCREOH study. Reproductive Health, 2021. 18(1): p. 136.

19. Chan, K.W., The household registration system and migrant labor in China: Notes on a debate. Population and development review, 2010. 36(2): p. 357-364.

20. Liu, X., et al., Factor structure of the 10-item perceived stress scale and measurement invariance across genders among Chinese adolescents. Frontiers in psychology, 2020. 11: p. 537. 
21. Cohen, S., T. Kamarck, and R. Mermelstein, Perceived stress scale. Measuring stress: A guide for health and social scientists, 1994. 10(2): p. 1-2.

22. Manea, L., S. Gilbody, and D. McMillan, A diagnostic meta-analysis of the Patient Health Questionnaire-9 (PHQ-9) algorithm scoring method as a screen for depression. General hospital psychiatry, 2015. 37(1): p. 67-75.

23. Wang, W., et al., Reliability and validity of the Chinese version of the Patient Health Questionnaire (PHQ-9) in the general population. General hospital psychiatry, 2014. 36(5): p. 539-544.

24. KC, B., H. MF, and B. SE, Intimate Partner Violence and Sexual Violence Victimization Assessment Instruments for Use in Healthcare Settings. 2007, Centers for Disease Control and Prevention National Center for Injury Prevention and Control: Atlanta (GA).

25. Wu, J., S. Guo, and C. Qu, Domestic violence against women seeking induced abortion in China. Contraception, 2005. 72(2): p. 117-121.

26. Funk, J.L. and R.D. Rogge, Testing the ruler with item response theory: increasing precision of measurement for relationship satisfaction with the Couples Satisfaction Index. Journal of Family Psychology, 2007. 21(4): p. 572-583.

27. Johnson, M.D., et al., Pathways to romantic relationship success among Chinese young adult couples: Contributions of family dysfunction, mental health problems, and negative couple interaction. Journal of Social and Personal Relationships, 2015. 32(1): p. 5-23.

28. Sherbourne, C.D. and A.L. Stewart, The MOS social support survey. Social science \& medicine, 1991. 32(6): p. 705-714.

29. Wang, W., et al., Psychometric testing of the Chinese Mandarin version of the Medical Outcomes Study Social Support Survey in patients with coronary heart disease in mainland China. Quality of Life Research, 2013. 22(8): p. 1965-1971.

30. Lai, J.C. and X. Yue, Using the Brief Resilience Scale to assess Chinese people's ability to bounce back from stress. Sage Open, 2014. 4(4): p. 2158244014554386.

31. Smith, B.W., et al., The brief resilience scale: assessing the ability to bounce back. International journal of behavioral medicine, 2008. 15(3): p. 194-200.

32. Fung, S.-f., Validity of the brief resilience scale and brief resilient coping scale in a Chinese sample. International journal of environmental research and public health, 2020. 17(4): p. 1265.

33. Field, A., Discovering statistics using IBM SPSS statistics. 2013: sage.

34. Ren, Y., et al., Public mental health under the long-term influence of COVID-19 in China: Geographical and temporal distribution. Journal of Affective Disorders, 2020. 277: p. 893-900.

35. Choi, E.P.H., B.P.H. Hui, and E.Y.F. Wan, Depression and Anxiety in Hong Kong during COVID-19. International Journal of Environmental Research and Public Health, 2020. 17(10): p. 3740.

36. Matud, M.P., Gender differences in stress and coping styles. Personality and Individual Differences, 2004. 37(7): p. 1401-1415. 
37. Olff, M., et al., Gender differences in posttraumatic stress disorder. Psychological bulletin, 2007. 133(2): p. 183.

38. Petch, J.F., et al., A randomized controlled trial of a couple relationship and coparenting program (Couple CARE for Parents) for high-and low-risk new parents. Journal of Consulting and Clinical Psychology, 2012. 80(4): p. 662-673.

39. Meyers-Levy, J. and B. Loken, Revisiting gender differences: What we know and what lies ahead. Journal of Consumer Psychology, 2015. 25(1): p. 129-149.

40. Joyce, S., et al., Road to resilience: a systematic review and meta-analysis of resilience training programmes and interventions. BMJ open, 2018. 8(6): p. e017858.

41. Cavallaro, F.L., et al., A systematic review of the effectiveness of counselling strategies for modern contraceptive methods: what works and what doesn't? BMJ sexual \& reproductive health, 2020. 46(4): p. 254-269.

42. Tiwari, A., et al., Effect of an advocacy intervention on mental health in Chinese women survivors of intimate partner violence: a randomized controlled trial. Jama, 2010. 304(5): p. 536-543.

43. Drew, L.B., et al., Intimate partner violence and effectiveness level of contraceptive selection postabortion. Journal of Women's Health, 2020. 29(9): p. 1226-1233.

44. Pallitto, C.C., et al., Intimate partner violence, abortion, and unintended pregnancy: results from the WHO Multi-country Study on Women's Health and Domestic Violence. International Journal of Gynecology \& Obstetrics, 2013. 120(1): p. 3-9.

45. Bradshaw, Z. and P. Slade, The effects of induced abortion on emotional experiences and relationships: A critical review of the literature. Clinical Psychology Review, 2003. 23(7): p. 929-958.

46. Ulloa, E.C. and J.F. Hammett, Temporal changes in intimate partner violence and relationship satisfaction. Journal of family violence, 2015. 30(8): p. 1093-1102.

47. Shortt, J.W., et al., The effects of intimate partner violence on relationship satisfaction over time for young at-risk couples: The moderating role of observed negative and positive affect. Partner abuse, 2010. 1(2): p. 131-151.

48. National Health Commission of the People's Republic of China, Report for COVID-19 Control and Prevention. 2021.

49. Singh, J. and J. Singh, COVID-19 and its impact on society. Electronic Research Journal of Social Sciences and Humanities, 2020. 2.

50. Ogrodniczuk, J.S., et al., Psychosocial impact of the COVID-19 pandemic: a cross-sectional study of online help-seeking Canadian men. Postgraduate Medicine, 2021: p. 1-10.

51. Bayefsky, M.J., D. Bartz, and K.L. Watson, Abortion during the Covid-19 pandemic-ensuring access to an essential health service. New England Journal of Medicine, 2020. 382(19): p. e47.

52. Luo, H., et al., Repeat induced abortion in 30 Chinese provinces: A cross-sectional study. International Journal of Gynecology \& Obstetrics, 2021. 
53. Zhuang, Y., X. Zhu, and L.-L. Huang, The effect of phloroglucinol on pain in first-trimester surgical abortion: a double-blind randomized controlled study. Contraception, 2010. 81(2): p. 157-160.

54. Ngo, T.D., M.H. Park, and Y. Xiao, Comparing the World Health Organization-versus Chinarecommended protocol for first-trimester medical abortion: a retrospective analysis. International journal of women's health, 2012. 4: p. 123.

55. Huang, Y., et al., A meta-analysis of the association between induced abortion and breast cancer risk among Chinese females. Cancer Causes \& Control, 2014. 25(2): p. 227-236.

\section{Supplementary Files}

This is a list of supplementary files associated with this preprint. Click to download.

- Additionalfile.docx 